\title{
Fermilab
}

\section{PRESSURE ANALYSIS OF CORRECTION COIL CRYOSTAT}

Eric Larson

May 28, 1982

The maximum working pressure of welded pressure vessels is commonly confirmed by test pressurizing to $125 \%$ and $150 \%$ of maximum working pressure in pneumatic and hydrostatic tests respectively. This demonstrates operation is possible at the MWP, but gives no measure of the maximum pressure the vessel can withstand without permanent deformation. By measuring the system bulk moduIus, $\beta_{s}$, of a fluid-filled vessel, the elastic limit can be explored without endangering the integrity of the vessel. The onset of nonlinearity in $\beta_{s}$ is a direct measure of the yield pressure of the weakest vessel component.

This procedure can be applied to vessels of unknown design origin and gire comparisons between actual and calculated strain when designing vessels. As will be shown, vessels which experience high pulsed pressures can also be tested in this fashion. In order to measure the bulk modulus of a vessel the volume expansion must be known as a function of pressure. One way to accomplish this with high resolution is to attach a sight gauge in series with the vessel and measure the fluid level as a function of pressure during a hydrostatic pressure test. The vessel must be free of gas which has a bulk modulus that varies with pressure, and can yield a correction factor which exceeds the measurement.

Small quantities of gas can be removed from the vessel after it is relatively full of water by drawing vacuum through the sight gauge and noting any gas bubbles which may appear. A change in the fluid level in the sight gauge shows the volume of gas displaced: The amount of trapped gas depends on the geometry of the vessel and the dissolved gases in the water.

Following this procedure the system may be pressurized at low pressures and a pressure vs volume plot recorded. Figure 1 shows a $P$ vs $V$ plot for a hypothetical pressure vessel. Curve \#I shows a small volume of air being pressurized. Curve $\# 2$ shows the elas- 
ticity of a hypothetical vessel. Curve \#3 shows the summation of the curves. The gas volume shows up as the $\Delta V$ between curve \#2 and Curve \#3. Figure 2 shows a plot of a vessel whose gas content is unknown. A line parallel to the linear portion of the curve which intersects the origin is shown dotted. The dotted line represents the $P$ vs $V$ plot for the vessel if there were no gas. Repeating the vacuum and pressurization techniques until the plot approaches linearity ensures the vessel is filled with water. The error that gas causes depends on the percentage of gas in the system, and the pressure range of the measurement.

Two smaller correction factors should be considered, the elasticity of the sight gauge, and the compressibility of water. The water correction becomes important only in large volume systems.

The bulk modulus of elasticity, $\beta$, for water is $3.2 \times 10^{5}$ psi at $60^{\circ} \mathrm{F}$ and $I \mathrm{~atm}$. Over the pressure range $0-3,000$ psi $\beta$ is constant.

$$
B=\frac{\Delta P}{\frac{\Delta V}{V}}
$$

where $\Delta P$ is a pressure change, $V$ is the total syster volume, and $\Delta V$ is the associated voiume change. Four thousand cubic inches of water pressurized to 100 psia would compress 1.25 cubic inches.

The elasticity of the sight gauge can be measured by pressurizing it alone and plotting $P$ vs $V$. Figure 3 shows a plot for the sight gauge used to test the cryostat on a correction spool piece. The gauge consists of an $8 \mathrm{ft}$ long - Lexan tube 1-3/4 in. $0 . \mathrm{d} . .$. 0.125 in. wall sealed with " $O$ " ring caps constrained by aluminum chennel.

By subtracting the gas, water, and sight gauge correction factors from the measurement, the true elasticity of the vessel can be cetermined.

This procedure was performed on a spool piece cryostat without a correction coil or beam tube. The cryostat was constrained to simulate a vacuum shield enclosure! The $1 \varnothing$ relief line was constrained at its end in a manner similar to an assembled spool piece. The flange at the base of the vacuum jacket for the power lead assembly was constrained as it would be when attached to the outer vacuum can of a spool. The ends of the 7-1/2 in. cryostat tube were closed with $1 / 8$ in. thick plate and reinforced with steel char- 
nel for rigidity. This was done to eliminate the end caps as a factor in the study.

The spacer bellows inside the power lead assembly was filled with water and sealed. It was noted that during pressurization the bellows would contract and cause volume changes which were not associated with the cryostat alone. By making the bellows essentially incompressible, the expansion of the cryostat could be studied alone.

Steps were then taken to ensure that all the gas was removed from the system before pressurization. Figure 4 and Table I show the data from the test. At pressures above 100 psig the cryostat begins to experience permanent deformation. After pressurizing the cryostat to $200 \mathrm{psig} 25$ cubic inches of permanent displacement was measured. Photos \#1 and \#2 show the cryostat bending due to the moment applied by the 1 in. $0 . d ., .049$ in. wall $2 \varnothing$ tube which runs parallel to the cryostat. The $2 \varnothing$ tube is welded to both end plates on the $1 \varnothing$ box and stiffens the box asymmetrically, causing a bending moment to occur as the opposite side of the box expands during pressurization. The moment leads to severe distortion at pressures above 220 psig and eventually contributed to the failure of the $1 \varnothing$ box. Failure eventualiy occurred at the weld connecting the upstream end plate to the top plate at 340 psig. The crack propagated over three sides quickly and completely separated the top plate from the lower front plate.

A second test was made with a fully assembled cryostat to measure the effect pressurization might have on the beam tube alignment. The $1 \varnothing$ bellows were constrained in a manner similar to tunnel installation and the power lead connections were closed with solder. Four alignment fixtures were placed inside the beam tube to monitor motion. The spool piece was set up for a hydrostatic test similar to the first test and cycled through several pressure ranges. Figure 5 and Table II show the $P$ vs $V$ data for this system. The location of the four fixtures and the direction of motion are shown in Table III. The motions of the alignment fixtures are shown referenced to their initial position. The important differences to note are the motions of the fixtures in relation to each other since their initial positions are arbitrary. To illustrate the motions of the alignment fixtures graphically the positions of the fixtures 
have been plotted against one another at various pressures, on Figure 6. All fixtures were initially referenced at 0,0 . 1,80 indicates the position of alignment of fixture \#1 at 80 psig; ail units of motion are in mils. Fixtures \#1, 2 and 4 seem to move predominantly along particular planes of motion.

Permanent deformation first occurs at 80 psig. At pressures above 130 psig creep deformation sets in as the plates on the 10 box attempt to form a more spherical shape. The beam tube was very stable, showing little motion and only slight hysteretic behavior at pressures below 100 psig.

The cryostat is supported at the four corners of the $1 \varnothing$ bottom plate, and the G-10 spacer ring on the cryostat tube in normal installation. The tested cryostat was supported at two corners of the bottom plate and the G-10 spacer ring, as shown in Figure 7 . This configuration facilitated leveling the cryostat and guaranteed contact at all three supports. The deformation of the bottom plate may have caused the vertical lifting seen in fixtures \#3 and \#4 at pressures above $100 \mathrm{psig.} \mathrm{This} \mathrm{could} \mathrm{also} \mathrm{explain} \mathrm{the} \mathrm{fact} \mathrm{that}$ all fixtures moved up from their initial positions.

An independert study of the deformation of the plates of the $2 \emptyset$ box was made during these pressure tests. A rigid platform was established to reference a dial indicator so the maximum plate deflection could be measured. The bottom plate was monitored on the cryostat without beam tube and the top plate was monitored on the fully assembled cryostat. Tables IV and $V$ show the data taken and compare it to theoretical deflections in two idealized cases. Case 36 for flat plates, from R.J.Roark's "Formulas for Stress and Strain", assumes the plate is simply supported on all edges. Case 41 assumes all edges of the plate are fixed. The plates on the $1 \varnothing$ box are neither of these two extremes, but instead a combination of the two cases. The larger the plate thickness is in relation to the weld cross section the more the plates act like the simply supported case. The maximum stress on each plate can be calculated and compared for each plate on the $1 \varnothing$ box, using Roark. The stress is a function of plate geometry, and the size of the applied load. Table VI shows a comparison of stresses on the six rectangular plates of the $1 \varnothing$ box. The end plates do not lend themselves to a simple stress analysis. The top and back plates have the highest stress concentrations, 
which explains the large deformations these plates experience in relation to the others. The stress factor can be cut to $2 / 3$ and the deflection almost in half if the thickness was $5 / 16$ in. instead of $1 / 4$ in.

Following the tests up to 240 psig the cryostat was pressurized to failure. The $1 \varnothing 3 / 4 \mathrm{in.} 0 . d$. tube which runs parallel to the beam tube separated from the upstream end plate on the $1 \varnothing$ box at 370 psig. The top, back and bottom plates on the $1 \varnothing$ box deformed considerably. The end flange on the $7-1 / 2$ in. $0 . d$. cryostat tube showed no visible deformation. The $1 \varnothing$ bellows and its squirm protectors were not deformed. The top plate on the power lead connectors and the soldered connections themselves showed no visible deformation. There was no other obvious weakness which could be concluded through visual inspection.

There did seem to be some pattern to the motion of the beam tube, but the exact cause of the motion is not determined. Certainly the asymmetric moment of the $2 \varnothing$ line was a contributing factor to these movements, but the extent to which it is responsible is as yet unknown. Other factors may contribute to, cancel, or dominate the motions detected. A great deal of effort was put intc immobilizing and isolating the cryostat during measurement, but accuracy can only be claimed within $\pm 2 \mathrm{mils}$ of the data.

Since the cryostat experiences peak pressures of 150 psi with 200. millisecond duration, a static test can only be used to give an indication of the cryostats performance under dynamic loading. In order to make a correlation between static and dynamic loading, further testing which simulates quench loads needs to be done. A quench might be simulated through two solenoid valves which could deliver and relieve pressure with a time function similar to a quench. Varying the magnitude of the pulse and noting if any permanent deformation occurred in the cryostat would establish a basis for comparing the two cases. 
(i)

(N)
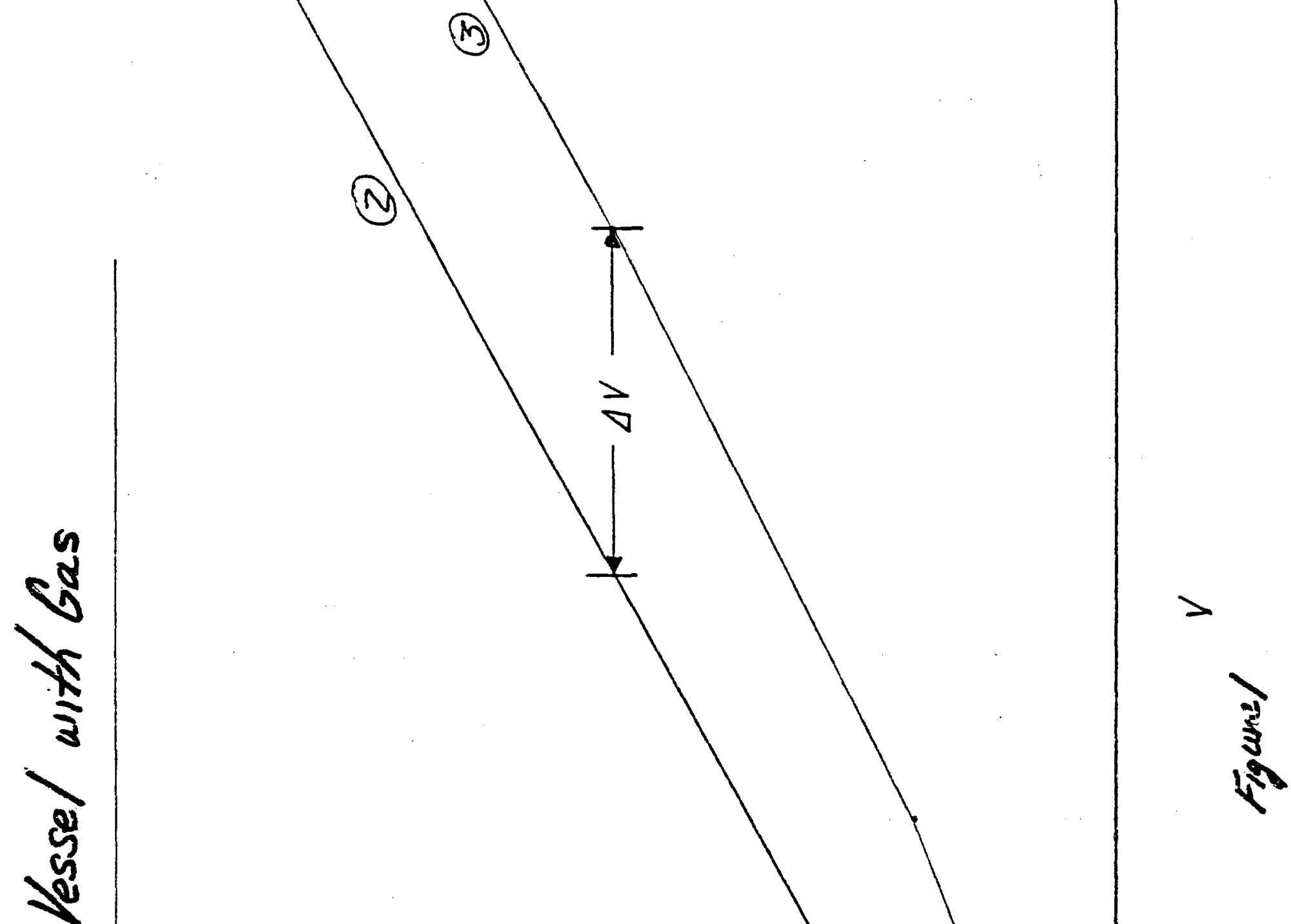

$>$

8

אר

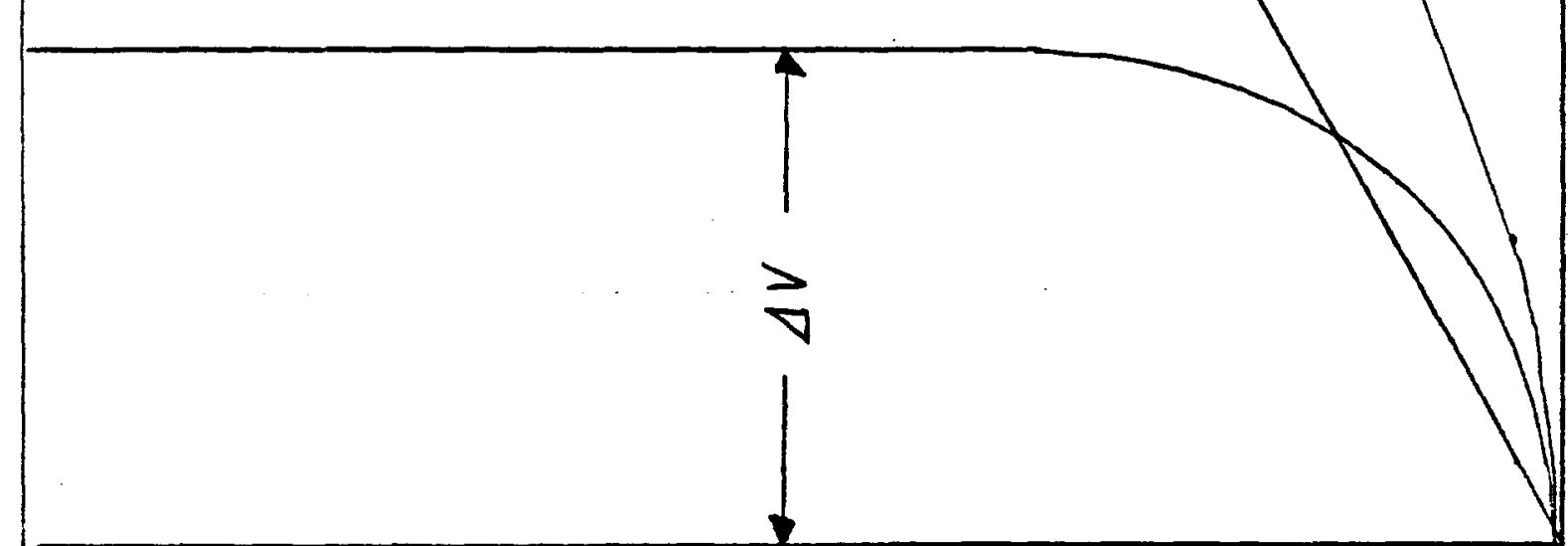

$\alpha$

$-6-$ 
Determination of Gas Volume

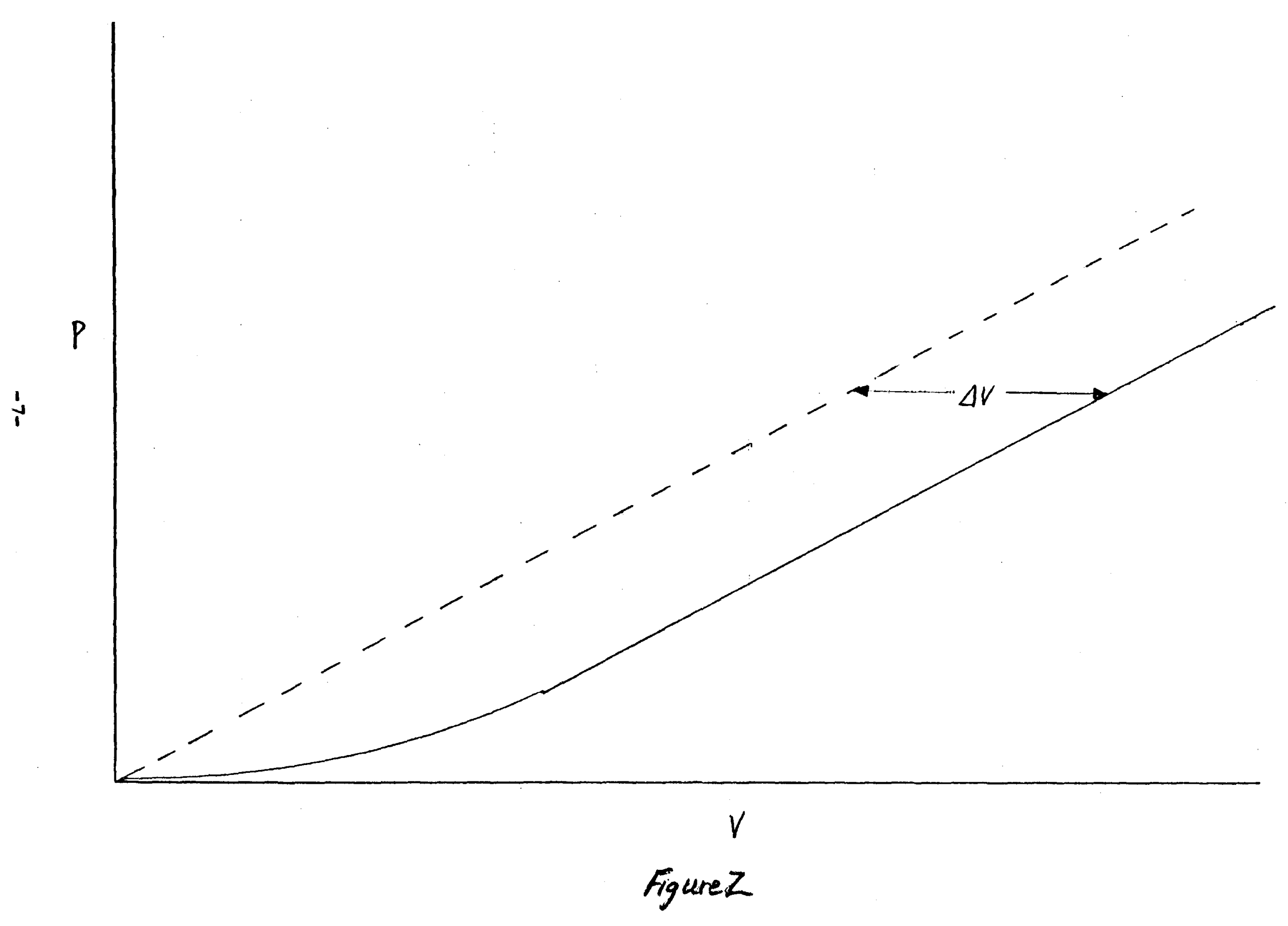

$\stackrel{\sum_{0}}{\stackrel{2}{*}}$ 


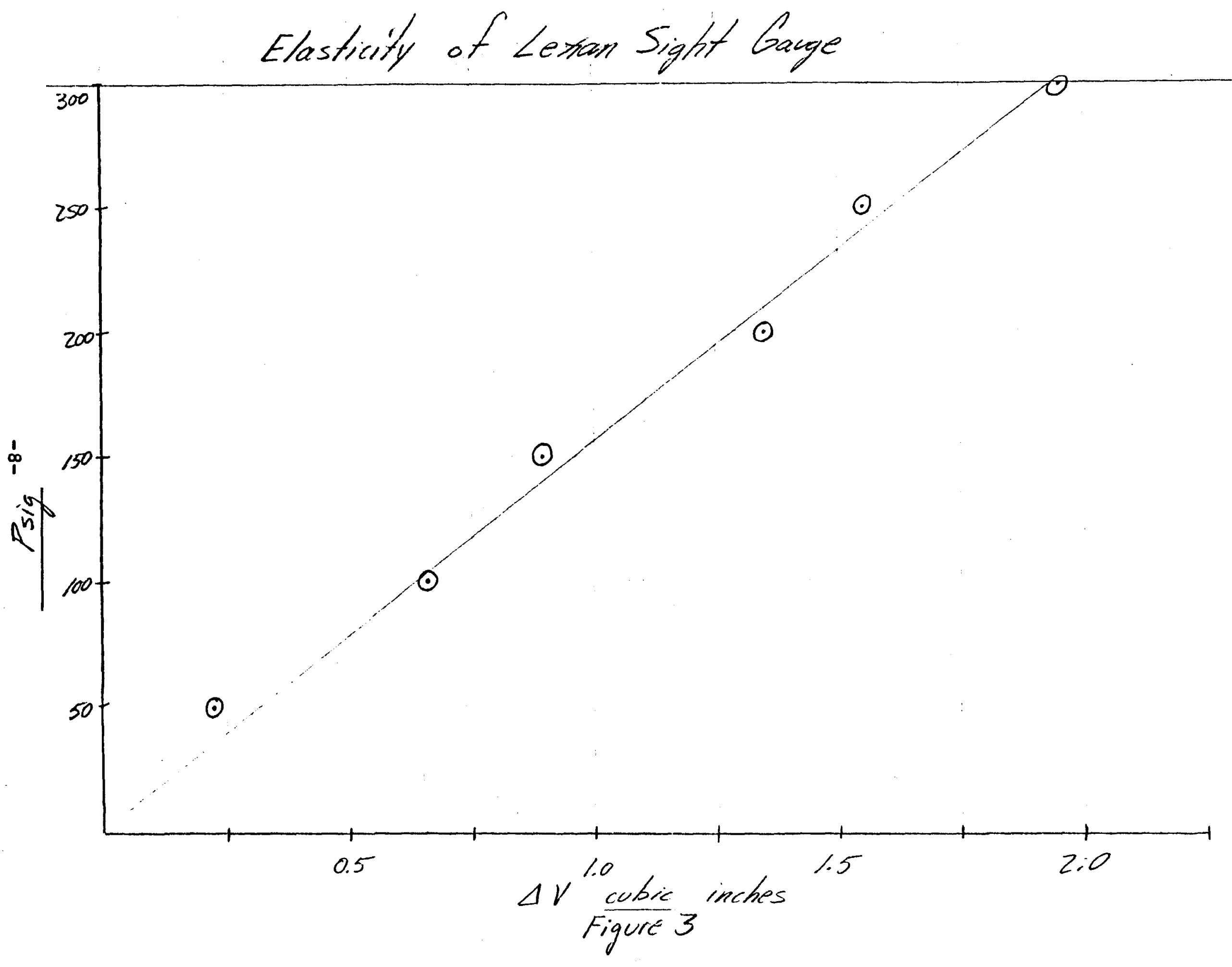

$\stackrel{1}{5}$ 


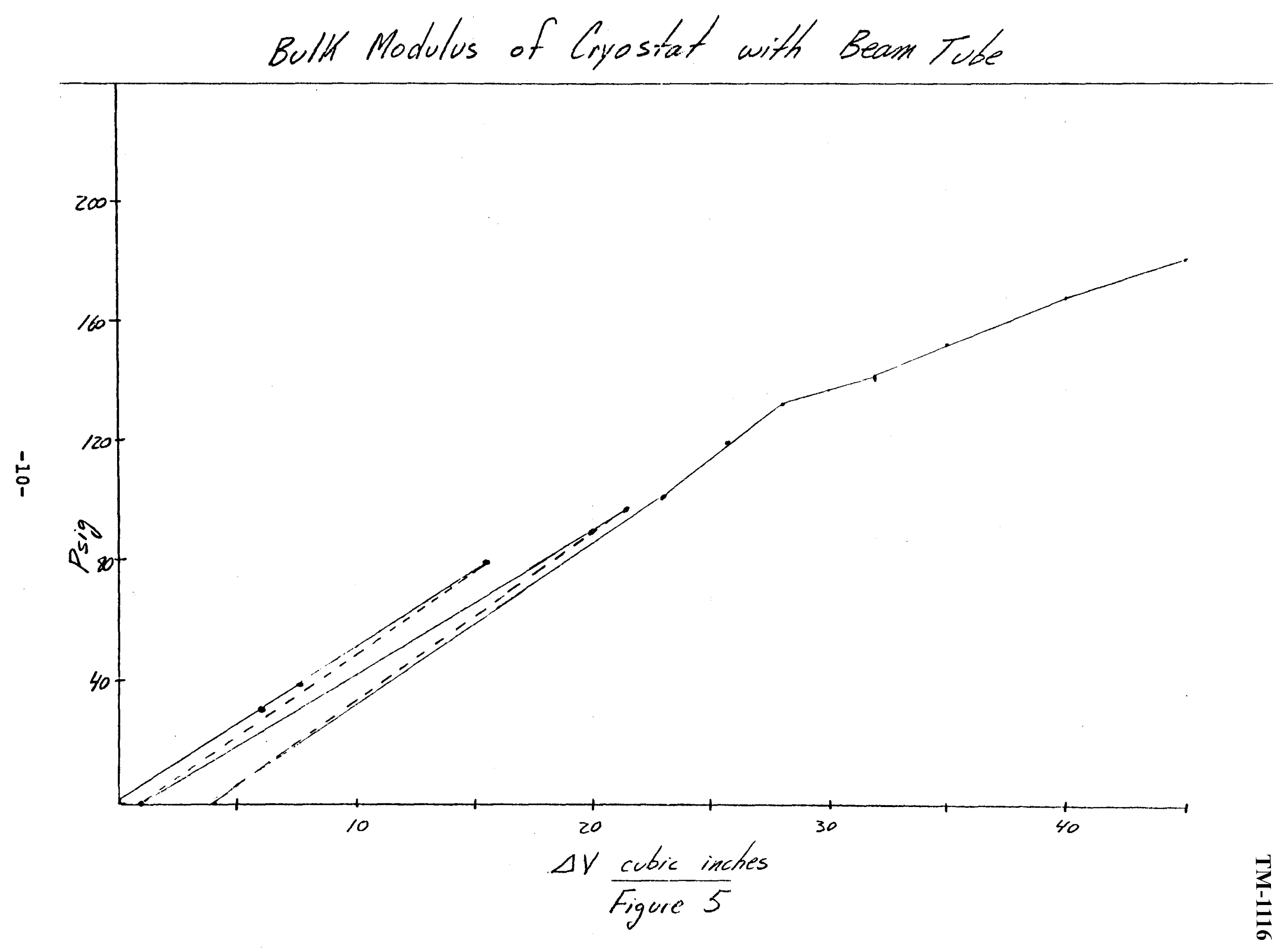




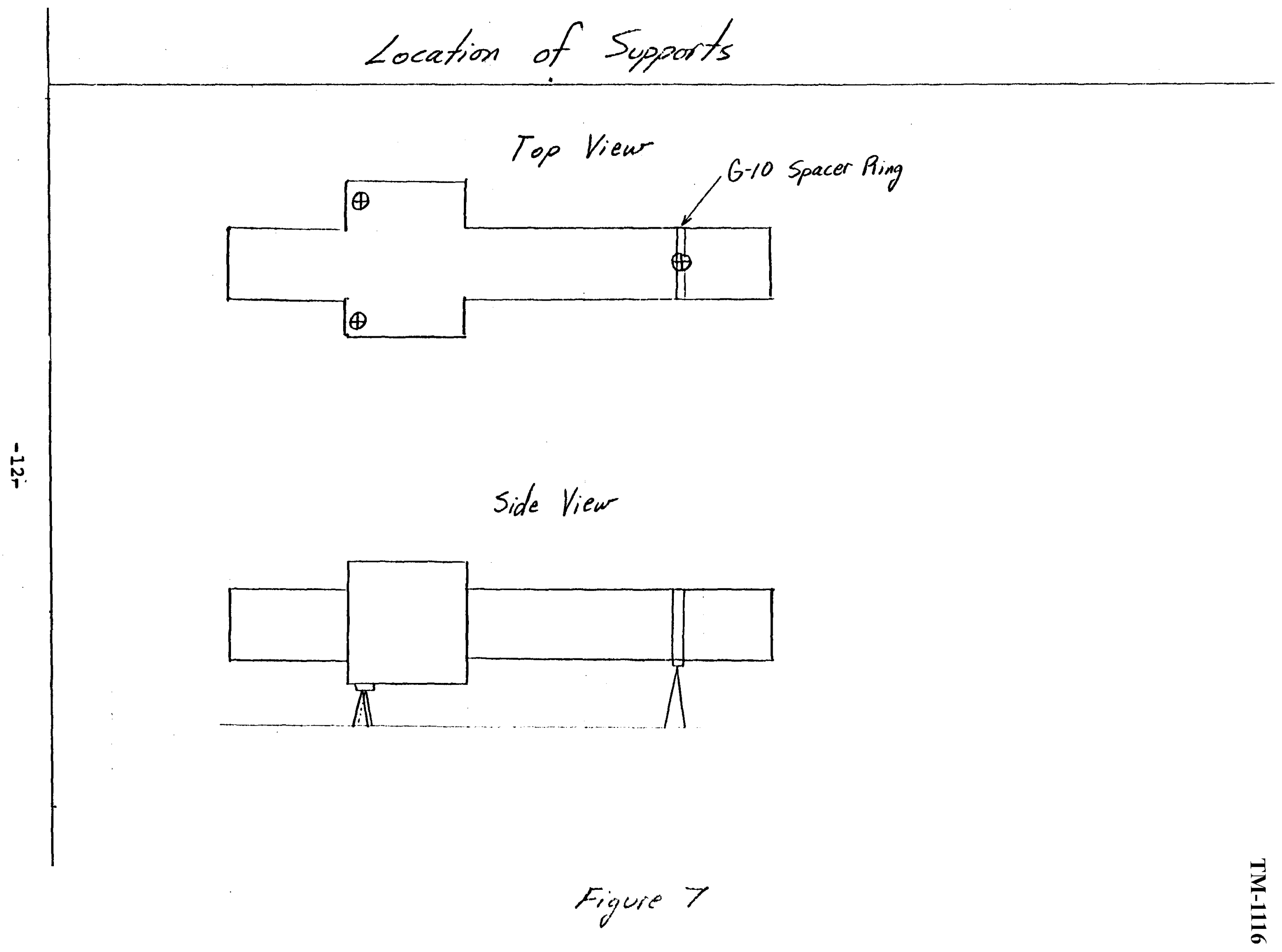




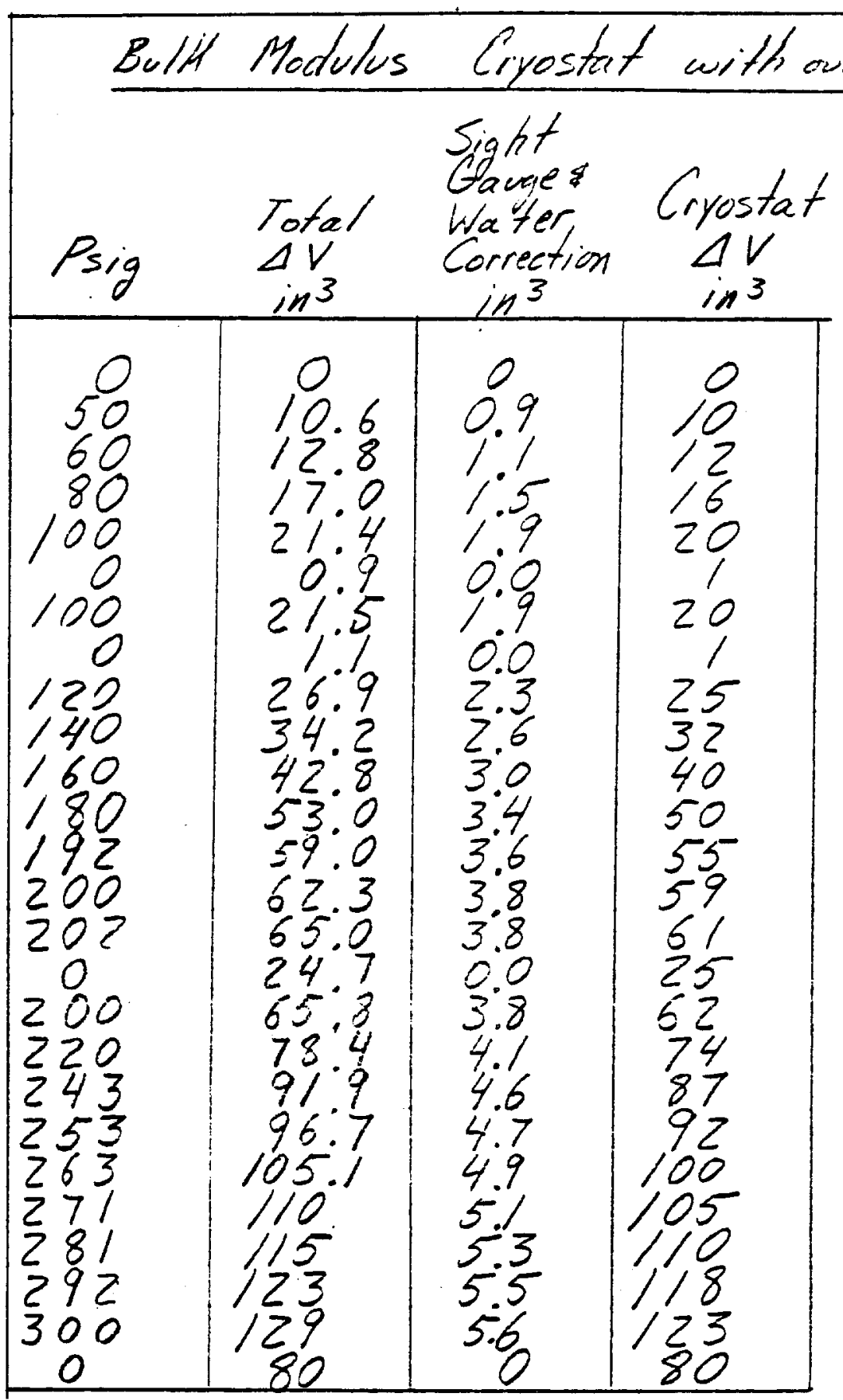




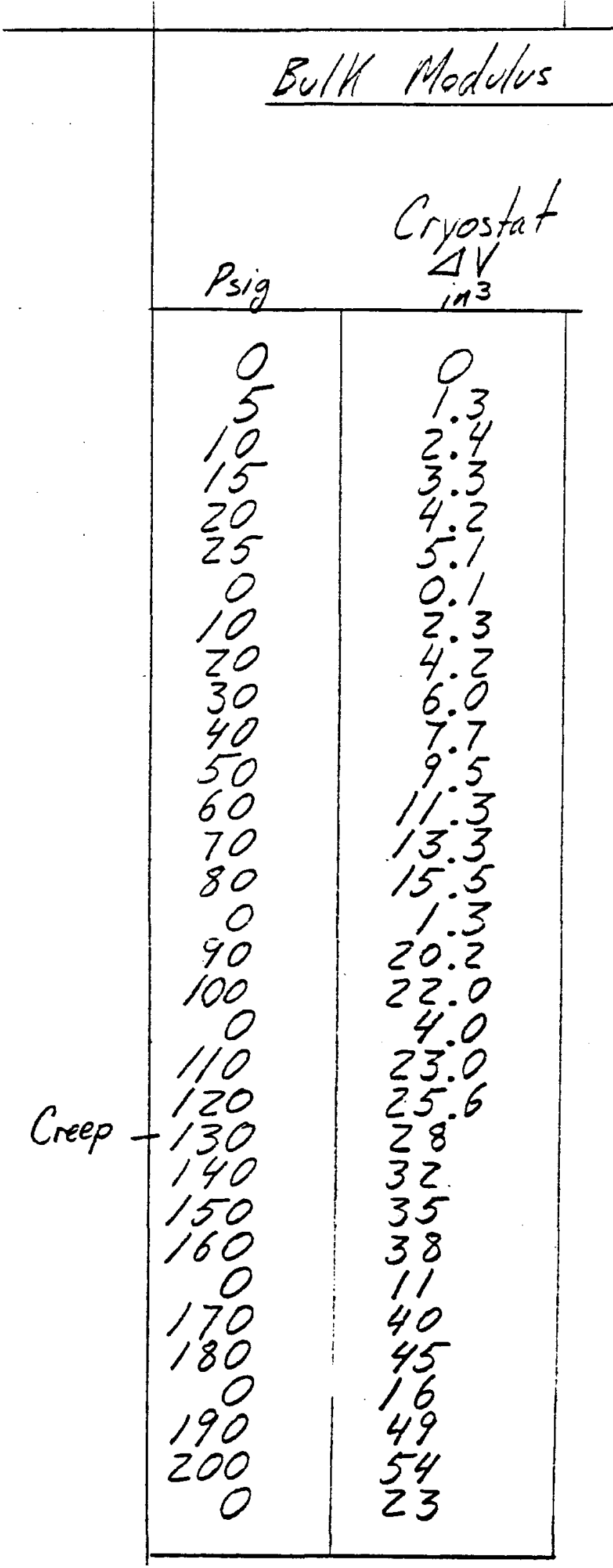

TABLE II 


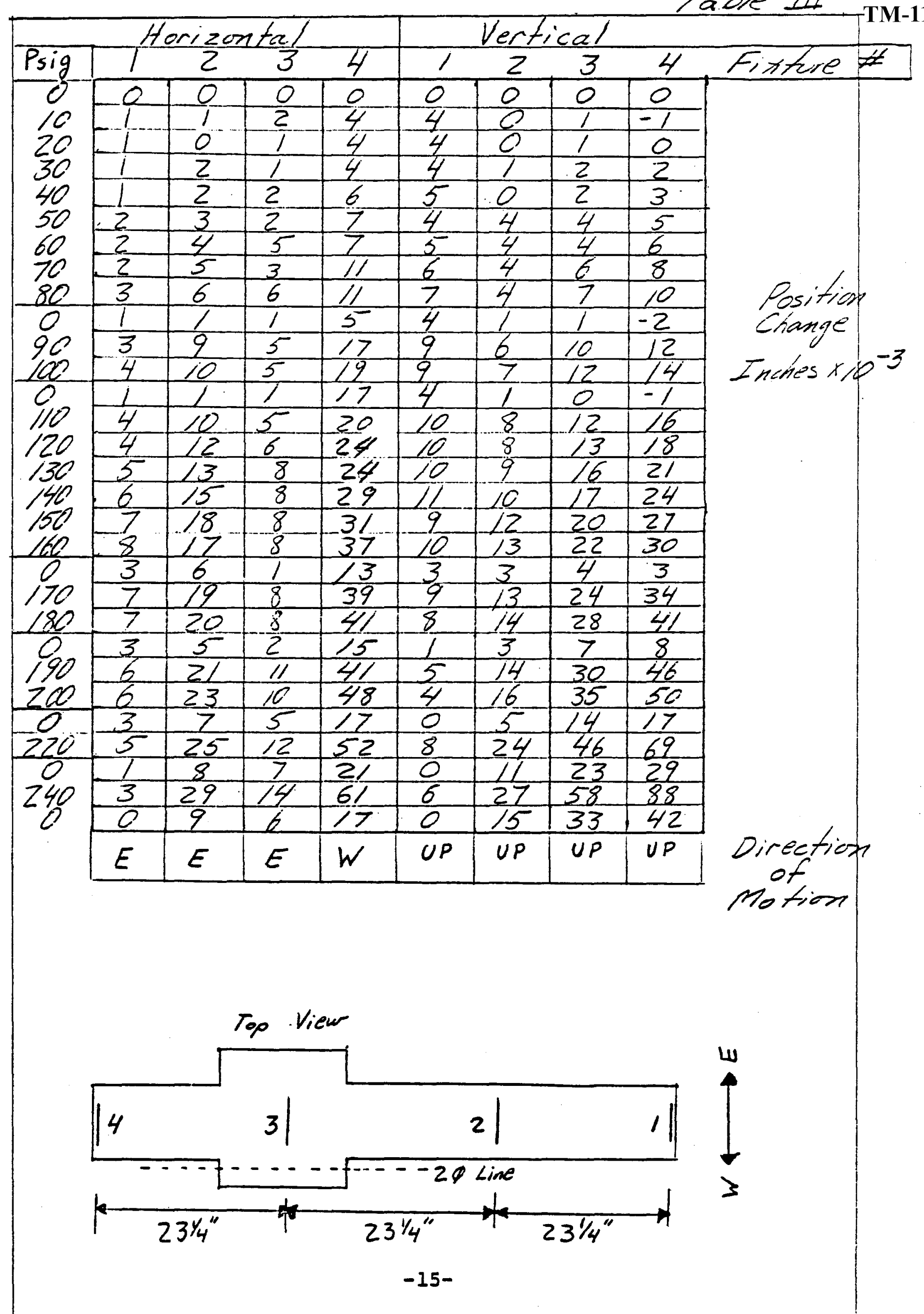




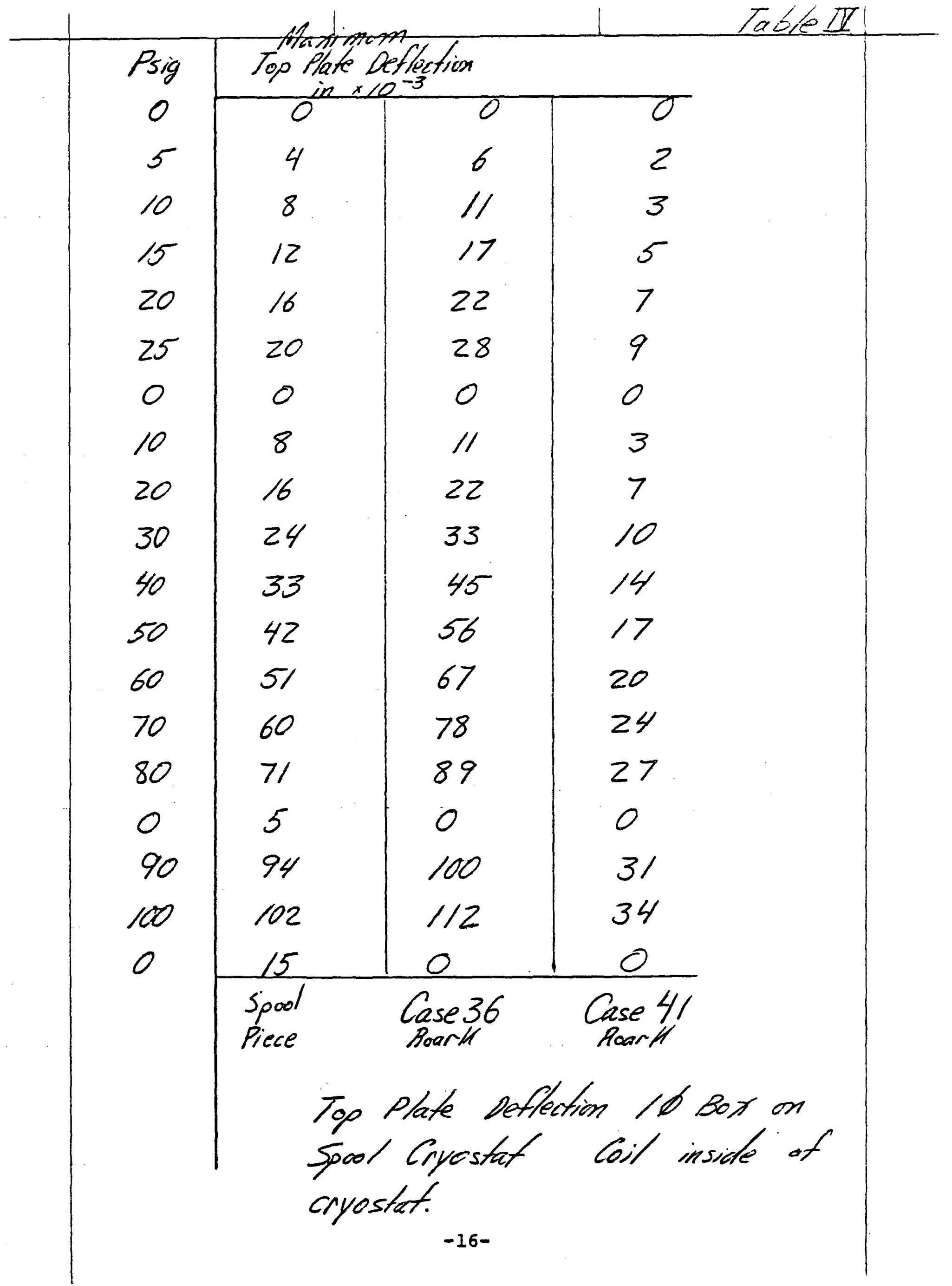


Table II

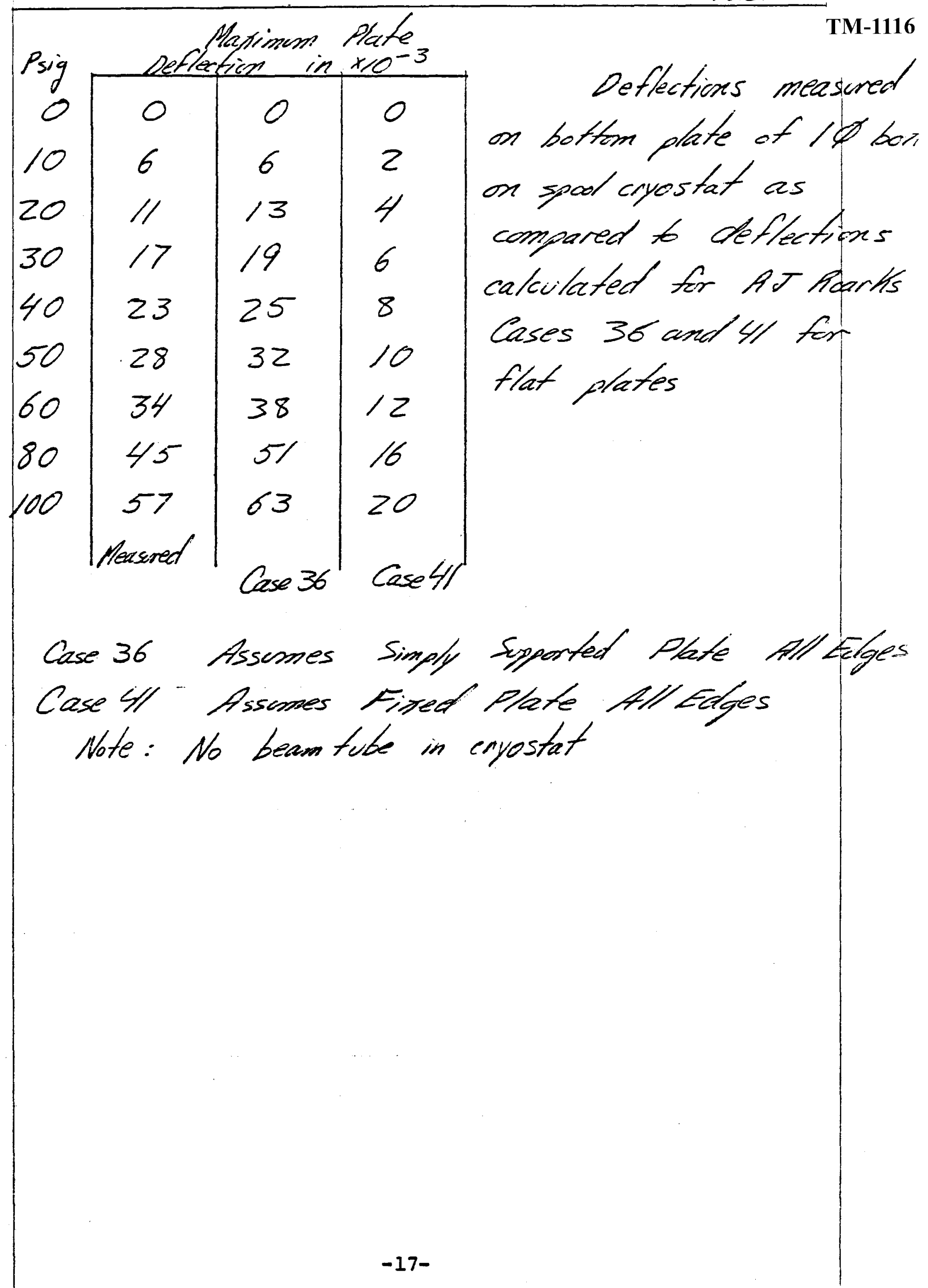




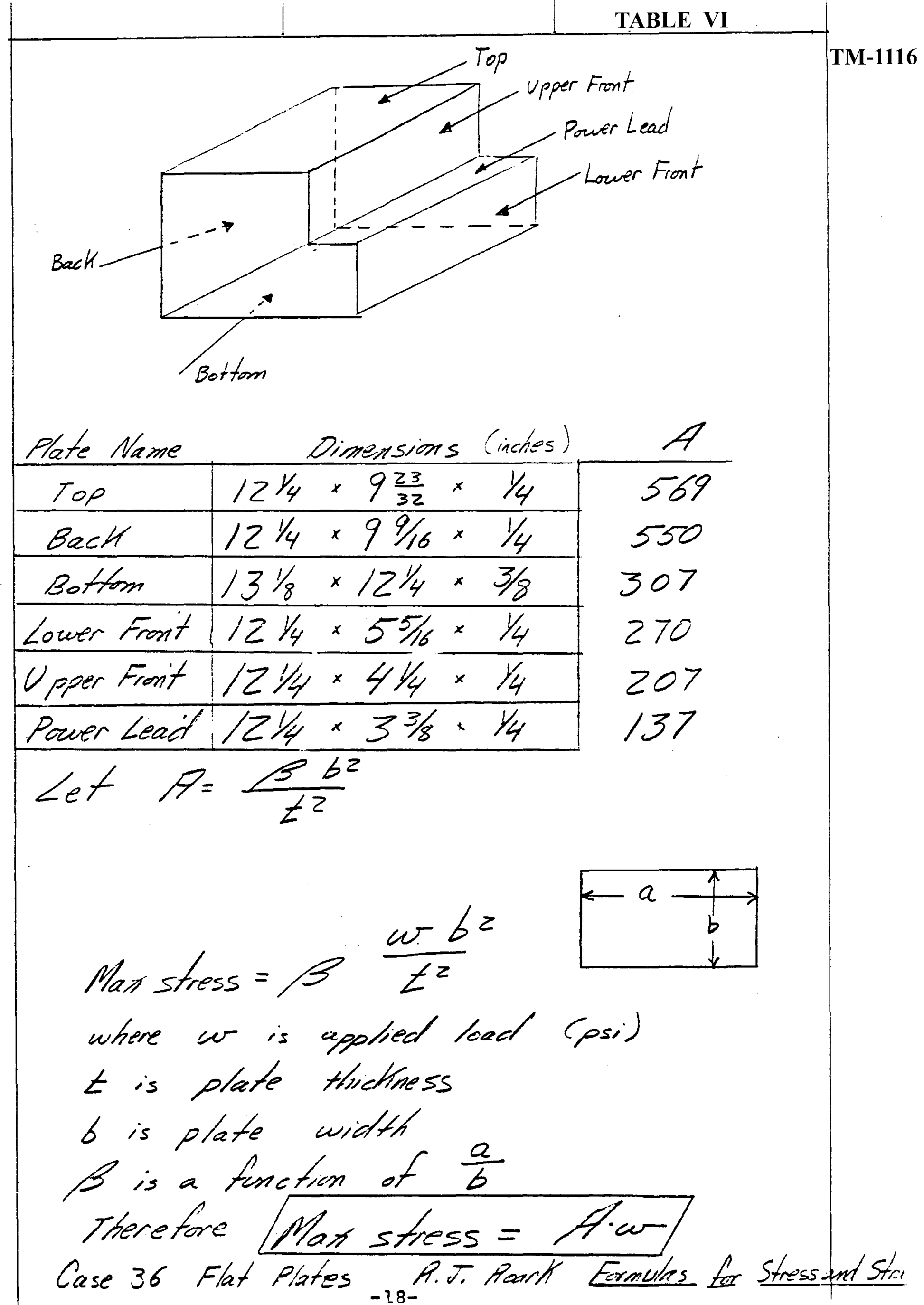




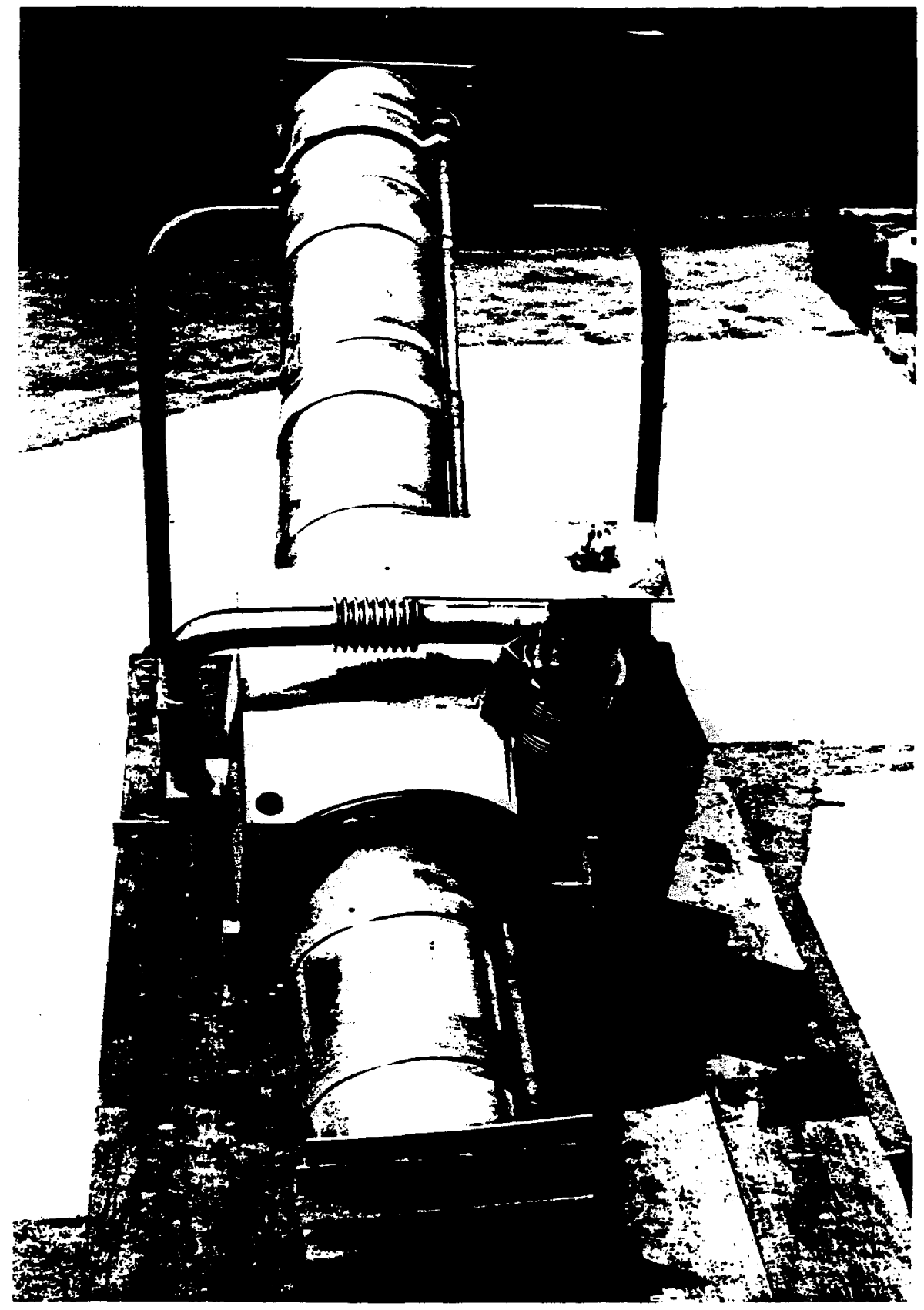

Photo \#1

Neg. \#82-222-7

$5 / 4 / 82$ 


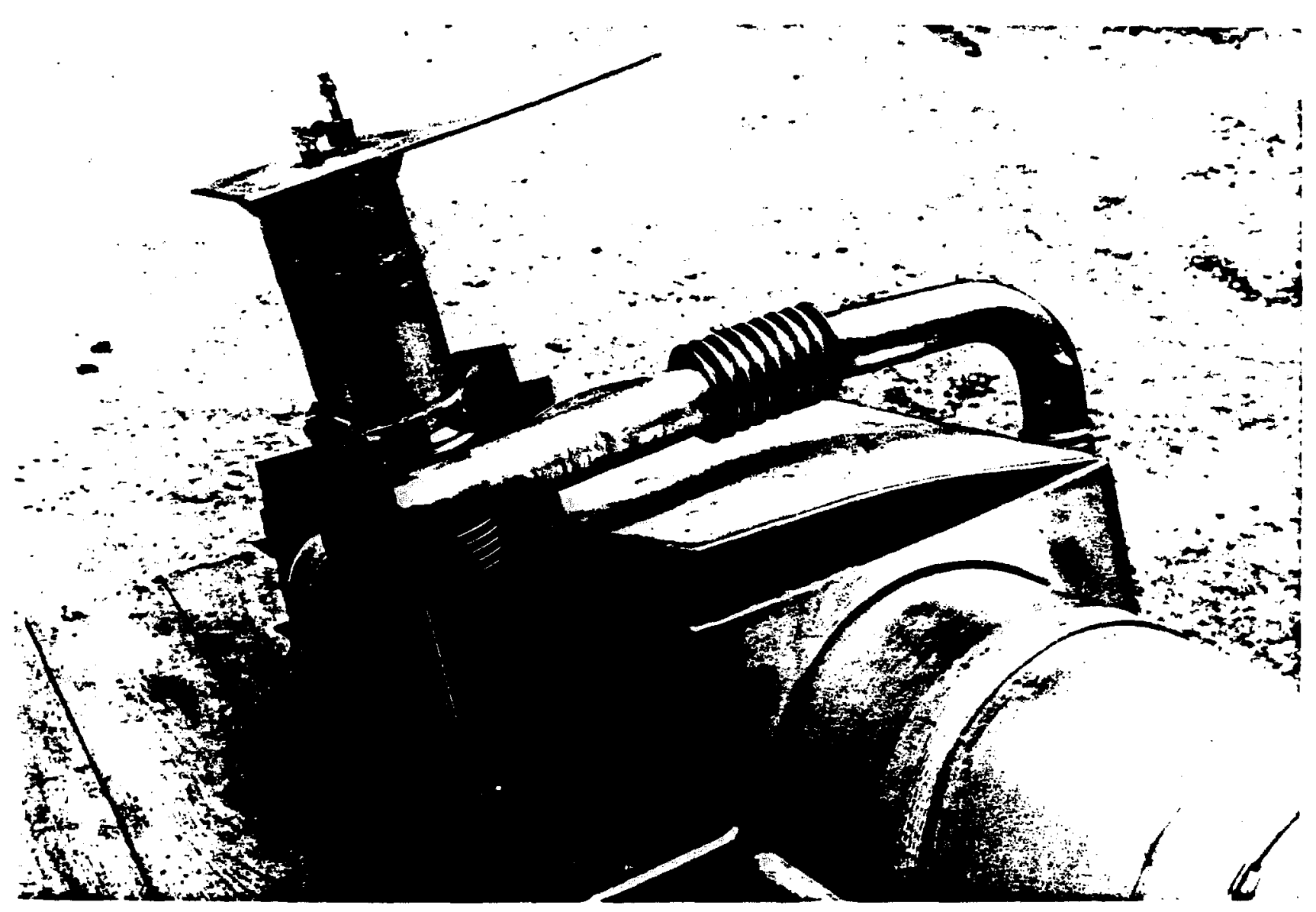

Photo \#2

Neg. \#82-222-5

$5 / 4 / 82$ 